\title{
Blue native DIGE as a tool for comparative analyses of protein complexes
}

Jesco Heinemeyer ${ }^{1}$, Burghardt Scheibe ${ }^{2}$, Udo K. Schmitz ${ }^{1}$, and Hans-Peter Braun ${ }^{1 *}$

${ }^{1}$ Institute for Plant Genetics, Faculty of Natural Sciences, Leibniz Universität Hannover, Herrenhäuser Str. 2, D-30419 Hannover, Germany

${ }^{2}$ GE Healthcare Europe GmbH, Oskar-Schlemmer-Str. 11, D-80807 München, Germany

* Corresponding author

Hans-Peter Braun

Institute for Plant Genetics

Leibniz Universität Hannover

Herrenhäuser Str. 2

D-30419 Hannover, Germany

Tel: +49 5117622674 , Fax: + 495117623608

e-mail: braun@genetik.uni-hannover.de

Key words: Differential gel electrophoresis, DIGE, Blue native PAGE, mitochondria, protein complexes, Arabidopsis thaliana

“DIGE”, “CyDye”, “Cy”, “DeCyder” and "Typhoon” are trademarks of GE Healthcare 


\section{Summary}

Differential gel electrophoresis (DIGE) is based on pre-labelling of different protein fractions and their subsequent co-electrophoresis in a single gel. Cyanine based "CyDye DIGE Fluor minimal dyes" are used for the labelling reaction and 2D IEF / SDS PAGE is the preferential electrophoresis system for protein separation. The DIGE technology allows elimination of inconsistencies based on gel to gel variations and furthermore allows exact quantification of proteins separated by gel electrophoresis. Here we report applications of the DIGE technology in combination with another 2D gel system, Blue native / SDS PAGE. "Blue native DIGE" offers (i) systematic and quantitative comparison of protein complexes of related protein fractions, (ii) structural investigation of protein complexes, (iii) assignment of protein complexes to subcellular fractions like organelles and (iv) electrophoretic mapping of isoforms of subunits of protein complexes with respect to a larger proteome. The potential of "Blue native DIGE" is illustrated by analysis of organellar fractions from the plant Arabidopsis thaliana and the alga Polytomella. Use of the DIGE technology for topological investigations is discussed. 


\section{Introduction}

Two-dimensional gel electrophoresis based on isoelectric focussing and SDS PAGE is a powerful technology for protein separation (O’Farrell 1975, Klose 1975). If applied under optimal conditions, more than 10000 different protein species can be resolved on single gels (Klose and Kobalz 1995). Even if carried out on medium-size gel systems, routinely more than 1000 proteins are separated. This makes 2D-PAGE an indispensable tool in protein research. However, two problems associated with this technology remained unsolved for quite a long time: insufficient reproducibility of gels and difficulties with respect to exact quantification of the proteins resolved on gels. Both were elegantly overcome about a decade ago by the invention of the 2D fluorescence differential gel electrophoresis (DIGE) (Ünlü et al. 1997, Tonge et al. 2001). DIGE is based on covalent labelling of proteins before electrophoresis by the cyanine dyes ("CyDye DIGE Fluor minimal dyes") Cy2, Cy3 and Cy5. The CyDyes are molecular weight matched, amine reactive and positively charged compounds. Based on substoichiometric labelling, no electrophoretic mobility shifts arise between differentially labelled samples upon co-electrophoresis. Two-dimensional DIGE allows the precise comparison of two or three proteome fractions within one gel, thereby eliminating inconsistencies due to technical gel to gel variations. Also, if substoichiometric labelling is applied, 2D DIGE allows reliable protein quantification. By using a pooled internal standard for DIGE experiments, relative protein quantification is highly accurate (Alban et al. 2003). For the last five years, several hundred successful differential proteome projects carried out on the basis of the DIGE technology have been published.

Besides IEF / SDS PAGE, other two-dimensional gel electrophoresis systems were developed which have very distinct properties. One especially successful "alternative" 2D system is Blue native / SDS PAGE (Schägger and von Jagow 1991). For this procedure, protein fractions are incubated with the anionic dye Coomassie-blue before electrophoresis. Coomassie gently introduces negative charge into proteins and protein complexes. In contrast to incubation with SDS, proteins remain native and protein complexes stable under the conditions applied. This offers to first resolve protein complexes in a native gel dimension. Afterwards, protein complexes are dissected into their subunits on a second gel dimension which is carried out in the presence of SDS. On the resulting 2D gels, proteins form characteristic vertical lines of 
spots. The proteins of one vertical line can be assumed to represent subunits of the same protein complex. Furthermore, 2D Blue native PAGE allows the analysis of hydrophobic membrane proteins, which are not separable by 2D IEF / SDS PAGE. It therefore became an important tool in membrane proteomics (Krause 2006).

Only a very limited number of publications so far report proteomic analyses based on Blue native PAGE and pre-labelling of protein fractions with fluorescent dyes (Perales et al. 2005, Granvogl et al. 2006, Reisinger and Eichacker 2007, Gillardon et al. 2007, Wittig et al. 2007, Dani and Dencher 2008). Here we show that combining 2D Blue native / SDS PAGE and the DIGE technology offers very special advantages for the comparative proteome analysis of membrane fractions and fractions containing protein complexes. Furthermore, DIGE in combination with Blue native PAGE allows to generate topological information on subcellular structures like organelles or protein complexes. Novel applications of Blue native DIGE are introduced and discussed.

\section{Materials and methods}

\section{$\underline{\text { Cultivation of plant material }}$}

Arabidopsis thaliana Ecotype Columbia (Col-O) was obtained from "The European Arabidopsis Stock Centre" (http://arabidopsis.info/). Cell suspension cultures were established according to May and Leaver 1993 and cultivated as outlined in Sunderhaus et al. 2006. Polytomella spp. (198.80, E.G. Pringsheim) was obtained from the „Sammlung von Algenkulturen der Universität Göttingen (SAG)“ (http://www.epsag.unigoettingen.de/html/sag.html). Cells were cultivated as described previously (Dudkina et al. 2005). 
Isolation of mitochondria and chloroplasts from plants

Isolation of mitochondria from Arabidopsis thaliana was carried out as given in Werhahn et al. 2001, isolation of chloroplast as outlined in Heinemeyer et al. 2004, and isolation of mitochondria from Polytomella as given in Dudkina et al. 2005.

\section{CyDye labeling of protein fractions prior to Blue native PAGE}

CyDye labeling of protein fractions was carried out as previously published (Perales et al. 2005). In brief: organelle fractions (each about $100 \mu \mathrm{g}$ protein) are centrifuged for 10 minutes at $4^{\circ} \mathrm{C}$ at full speed using an Eppendorf centrifuge. Sedimented organelles are re-suspended in $10 \mu 1$ "Solubilization buffer $\mathrm{pH} 7.4$ + detergent" (30 mM HEPES, $150 \mathrm{mM}$ potassium acetate, $10 \%$ [v/v] glycerol, $\mathrm{pH} 7.4$, supplemented with $1 \%$ dodecylmaltoside or 5\% Digitonin [solution should be briefly boiled to get Digitonin dissolved]). Resulting amount detergent / protein is $1 \mathrm{~g} / \mathrm{g}$ for dodecylmaltoside and $5 \mathrm{~g} / \mathrm{g}$ for digitonin. Fractions are again centrifuged for 20 minutes at $4^{\circ} \mathrm{C}$ at full speed using an Eppendorf centrifuge to remove insoluble material. The supernatant containing solubilized protein complexes is supplemented with $10 \mu 1$ "Solubilization buffer $\mathrm{pH}$ 10" (30 mM HEPES, $150 \mathrm{mM}$ potassium acetate, 10\% [v/v] glycerol) to adjust the $\mathrm{pH}$ value of the protein solution to about $\mathrm{pH} 8.5$, which is a prerequisite for efficient labeling. One $\mu 1$ "CyDye working solution" (400 pmol/ $\mu 1$ reconstituted CyDyes in DMF prepared according to manufacturer's instructions, GE Healthcare, Munich, Germany) is added to the protein fraction. Labeling takes place for 10 minutes in the dark and is stopped by addition of $1 \mu \mathrm{l}$ "Lysine solution" (10 mM lysine). Finally, the sample is supplemented with $1 \mu 1$ "Blue loading buffer" (750 mM aminocaproic acid, $5 \%[\mathrm{w} / \mathrm{v}$ ] Coomassie $250 \mathrm{G}$ ) and directly transferred into the slot of a blue native gel.

\section{Gel electrophoresis procedures and electroelution}

Protein separations by two-dimensional Blue native / SDS PAGE followed the protocol given by Wittig et al. 2006. Three-dimensional Blue native / IEF / SDS PAGE was carried out as given in Werhahn and Braun 2002. Image acquisition was performed with Typhoon TRIO and quantification of the CyDye labelled proteins resolved by 2D Blue native PAGE was carried out using the DeCyder 2D software (both GE Healthcare, Munich, Germany). Quantifications should be based on biological replicates. 


\section{Results and Discussion}

In contrast to a standard DIGE experiment followed by protein separation using IEF / SDS PAGE, pre-labeling of protein fractions before separation by Blue-native PAGE takes place under native conditions. Therefore, protein fractions should include low salt concentrations and membranes should be solubilized by very gentle non-ionic detergents. For our investigation, $1 \mathrm{~g}$ dodecylmaltoside / $\mathrm{g}$ protein or $5 \mathrm{~g}$ digitonin / g protein proved to yield good results. Protein solubilization is carried out at $\mathrm{pH} 7$, but labeling at a more basic $\mathrm{pH}$ for increasing the efficiency of the labeling reactions (details are given in the Material and methods section). Using this experimental strategy, "Blue native DIGE" allows several applications for proteomics:

\section{$\underline{1 . \text { General comparison of related protein fractions }}$}

The basic application of DIGE in combination with 2D IEF / SDS PAGE is the comparison of related protein fractions of individuals differing in developmental or health state, individuals differing in some kind of pre-treatment or individuals exhibiting genotypic differences. "Blue native DIGE” allows proteomic analysis in a similar way but rather visualizes differences concerning hydrophobic proteins and / or protein complexes. The very first published application of Blue native DIGE was dedicated to a comparison of a wild-type Arabidopsis line and a corresponding Arabidopsis knock-out line that lacks a gene encoding a newly discovered carbonic anhydrase present within mitochondrial complex I of plants (Figure 1; Perales et al. 2005). Analysis by "Blue native DIGE" revealed drastically reduced levels of mitochondrial complex I and a complex I including respiratory supercomplex in the mutant line (green rows of spots on the gel shown in Figure 1). Evaluation based on the DeCyder 2D software consistently revealed about 8 fold reduction of complex I subunits in mutant Arabidopsis plants (Perales et al. 2005). In general, differential abundance of protein complexes in two protein fractions to be compared nicely can be analyzed by "Blue native DIGE”. 


\section{$\underline{\text { 2. Comparison of differentially treated protein fractions }}$}

Protein complexes differ with respect to stability. For instance, heat, salt or detergent treatment easily can lead to dissociation of protein complexes. On the other hand, controlled destabilization of protein complexes into subcomplexes can give interesting insights into the architecture of large protein particles. Corresponding experiments can be evaluated using "Blue native DIGE". The heat stability of the protein complexes constituting the mitochondrial Oxidative Phosphorylation (OXPHOS) system was investigated by differential labelling of a heat-treated and a control fraction that was kept on ice (Figure 2). Blue native DIGE nicely reveals dissociation of mitochondrial complexes I, II and IV but stable complexes $\mathrm{III}_{2}$ and $\mathrm{V}$ under the conditions applied. Stability of membrane inserted protein complexes may not only be influenced by interaction of their subunits but also by the developmental stage and the lipid content of the membrane (Kota et al. 2002). There are a variety of parameters which may be changed in the type of experimental setup illustrated in Fig. 2. If analysed by the DeCyder software, destabilization of protein complexes can be exactly quantified.

\section{$\underline{\text { 3. Comparison of proteomes from different organisms }}$}

The subunit composition of protein complexes often is compared between different species to understand differences in function and activity or to follow molecular evolution. "Blue native DIGE" offers to precisely compare protein complex fractions of different organisms without interference of gel to gel variations. As an example, mitochondrial membrane fractions of Arabidopsis thaliana and the alga Polytomella were differentially labelled with CyDyes and compared (Figure 3). The following results were obtained: The protein complexes of the OXPHOS complexes of Polytomella (given in red on the Figure 3) are slightly smaller than the same complexes from Arabidopsis (see for example complexes I and $\mathrm{III}_{2}$ ). This either could be due to absence of subunits in the OXPHOS complexes of Polytomella, or to a smaller size of proteins of the same set of subunits. In the case of complex III, nearly all visible subunits of Polytomella indeed are slightly smaller. Comparing protein complex quantities, complex I of Polytomella clearly is of lower abundance if compared to Arabidopsis. One further difference between the OXPHOS system of Polytomella and Arabidopsis concerns its supramolecular organisation. While ATP synthase dimers are known 
to be extremely stable in Polytomella upon solubilization of mitochondria using dodecylmaltoside, these dimers are dissected into monomers in Arabidopsis. In conclusion, "Blue native DIGE" nicely allows comparison of protein complexes of related membranes from different organisms.

\section{Comparison of different subcellular proteomes}

Furthermore, "Blue native DIGE" offers to study different subcellular proteomes of an organism within one single gel, e.g. the proteomes of different organelles. This is illustrated by comparing differentially labelled protein complexes of mitochondria and chloroplasts of Arabidopsis (Figure 4). On the figure, the subunits of the mitochondrial OXPHOS system are represented by green and the chloroplast protein complexes involved in photosynthesis by red spots. This Blue native DIGE approach has several applications. First of all, fractions of isolated organelles often are cross-contaminated by other types of organelles. If three fractions of partially purified organelles - e.g. enriched chloroplasts, mitochondria and peroxisomes are differentially labelled, mixed, and subsequently analysed by "Blue native DIGE", the colour of a complex allows its assignment to a fraction of highest enrichment. This greatly facilitates assignment of newly described protein complexes to subcellular fractions. Also, 2D Blue native / SDS gels showing protein complexes CyDye-labelled according to subcellular localization may serve as reference gels to evaluate cross-contaminations of purified organelle fractions. Similar applications of the DIGE system were previously reported also for the 2D IEF / SDS PAGE system: if organelle fractions of increased purity (e.g. successive fractions taken from the steps of an organelle preparation) are differentially labelled with CyDyes, relative down-concentration of protein species becomes visible by colour which allows to identify them as contaminations with respect to the organelle fraction of interest (Eubel et al. 2007, Eubel et al. 2008, Lee et al. 2008).

\section{Three-dimensional Blue native / IEF / SDS PAGE}

A three dimensional Blue native / IEF / SDS PAGE system was suggested to separate isoforms of subunits of protein complexes (Werhahn and Braun 2002). Starting point of the procedure are electroeluted protein complexes separated on a 1D Blue native gel which 
subsequently are separated by two further gel dimensions using IEF and SDS-PAGE. For example, most subunits of the OXPHOS complexes are present in multiple isoforms in higher eukaryotes like vertebrates or plants. These nicely can be mapped on the basis of the suggested gel system. Three-dimensional Blue native / IEF / SDS PAGE also elegantly can be realized on the basis of the DIGE technology. For this approach, two electroeluted protein complexes are differentially pre-labelled with two CyDyes. A third CyDye can be used to label a reference proteome, which is used as a background. Finally, all thee fractions are mixed, co-electrophoresed on one gel and proteins are detected at the respective wavelengths using the Typhoon scanner (Figure 5). As an example, mitochondrial complex I and V from Arabidopsis as well as the total mitochondrial proteome are differentially labelled and analysed by the DIGE technology (Figure 6). On the resulting 3D gel, the subunits of complex $\mathrm{I}$ and $\mathrm{V}$ including postranslationally modified versions and isoforms are visible in blue and green in front of the total mitochondrial proteome given in red. This type of three dimensional separation systematically allows electrophoretic mapping of proteins forming part of protein complexes within a defined cellular fraction.

\section{$\underline{\text { 6. Topological characterization of protein complexes }}$}

Finally, the CyDyes also have a potential to reveal topological information. Various procedures were suggested to use surface labelling of native structures like organelles or protein complexes to identify surface exposed components, e.g. based on biotin labelling (Shin et al. 2003). This approach basically also could work using the CyDyes. It previously was reported that labelling of intact animal cells by one CyDye followed by labelling of all proteins after cell lysis with another CyDye allows to identify proteins exposed on the cell surface (Hagner-McWhirter et al. 2004). Attempts to deduce topological information using the CyDyes were also reported for studying the photosystems of Arabidopsis (Granvogl et al. 2006). However, reliable labelling of surface exposed subunits of the photosystem complexes was not observed. In contrast, in our hands, differential labelling of surface exposed proteins of organelles and protein complexes proved to be possible if the CyDyes used for labelling are diluted and if the labelling reaction takes place for very short time periods in the range of seconds (data not shown). Optimization of suitable protocols is under way in our laboratory. 
In summary, combining CyDye labelling and "Blue native PAGE" offers numerous novel applications which should be useful for future proteome research.

\section{Acknowledgments}

We thank Professor Lutz Eichacker, Stavanger University, Norway, for advice and helpful discussions. This research was supported by the Deutsche Forschungsgemeinschaft (grant $\mathrm{Br}$ $1829-7 / 3)$.

\section{References}

Alban A, David SO, Bjorkesten L, Andersson C, Sloge E, Lewis S, Currie I. A novel experimental design for comparative two-dimensional gel analysis: two-dimensional difference gel electrophoresis incorporating a pooled internal standard. Proteomics 2003; 3:36-44.

Dani D, Dencher NA. Native-DIGE: a new look at the mitochondrial membrane proteome. Biotechnol J. 2008;3:817-822.

Dudkina NV, Heinemeyer H, Keegstra W, Boekema EJ, Braun HP. Structure of dimeric ATP synthase from mitochondria: An angular association of monomers induces the strong curvature of the inner membrane. FEBS Lett. 2005;579:5769-5772.

Eubel H, Lee CP, Kuo J, Meyer EH, Taylor NL, Millar AH. Free-flow electrophoresis for purification of plant mitochondria by surface charge. Plant J. 2007;52:583-594

Eubel H, Meyer EH, Taylor NL, Bussell JD, O'Toole N, Heazlewood JL, Castleden I, Small ID, Smith SM, Millar AH. Novel proteins, putative membrane transporters and an integrated metabolic network are revealed by quantitative proteomic analysis of Arabidopsis cell culture peroxisomes. Plant Physiol. 2008; in press

Gillardon F, Rist W, Kussmaul L, Vogel J, Berg M, Danzer K, Kraut N, Hengerer B. Proteomic and functional alterations in brain mitochondria from $\mathrm{Tg} 2576$ mice occur before amyloid plaque deposition. Proteomics 2007;7:605-616 
Granvogl B, Reisinger V, Eichacker LA. Mapping the proteome of thylakoid membranes by de novo sequencing of intermembrane peptide domains. Proteomics 2006;6:3681-3695.

Hagner-McWhirter A, Bourin S, Nejad MR, Marounga R. Selective labelling of cell surface proteins with CyDye DIGE Fluor minimal dyes. Application Note 2004; GE Healthcare

Heinemeyer J, Eubel H, Wehmhöner D, Jänsch L, Braun HP. Proteomic approach to characterize the supramolecular organization of photosystems in higher plants. Phytochemistry 2004;65:1683-1692.

Klose J. Protein mapping by combined isoelectric focusing and electrophoresis of mouse tissues. A novel approach to testing for induced point mutations in mammals. Humangenetik. 1975;26:231-43

Klose J, Kobalz U. Two-dimensional electrophoresis of proteins: an updated protocol and implications for a functional analysis of the genome. Electrophoresis 1995;16:1034-1059.

Kota Z, Horvath LI, Droppa M, Horvath G, Farkas T, Pali T. Protein assembly and heat stability in developing thylakoid membranes during greening. PNAS 2002;12149-12154

Krause F. Detection and analysis of protein-protein interactions in organellar and prokaryotic proteomes by native gel electrophoresis: (Membrane) protein complexes and supercomplexes. Electrophoresis. 2006;27:2759-2781.

Lee CP, Eubel H, O'Toole N, Millar AH. Heterogeneity of the mitochondrial proteome for photosynthetic and non-photosynthetic Arabidopsis metabolism. Mol Cell Proteomics. 2008;7:1297-1316

May MJ, Leaver CJ. Oxidative stimulation of glutathione synthesis in Arabidopsis thaliana suspension cultures. Plant Physiol. 1993;103:621-627.

O'Farrell PH. High resolution two-dimensional electrophoresis of proteins. J Biol Chem. 1975;250:4007-21.

Perales M, Eubel H, Heinemeyer J, Colaneri A, Zabaleta E, Braun HP. Disruption of a nuclear gene encoding a mitochondrial gamma carbonic anhydrase reduces complex I and supercomplex I+III2 levels and alters mitochondrial physiology in Arabidopsis. J. Mol. Biol. 2005;350:263-277.

Reisinger V, Eichacker LA. How to analyze protein complexes by 2D blue native SDSPAGE. Proteomics. 2007; Suppl 1:6-16

Schägger H, von Jagow G. Blue native electrophoresis for isolation of membrane protein complexes in enzymatically active form. Anal Biochem. 1991;199:223-231. 
Shin BK, Wang H, Yim AM, Le Naour F, Brichory F, Jang JH, Zhao R, Puravs E, Tra J, Michael CW, Misek DE, Hanash SM. Global profiling of the cell surface proteome of cancer cells uncovers an abundance of proteins with chaperone function. J Biol Chem. 2003;278:7607-7616

Sunderhaus S, Dudkina N, Jänsch L, Klodmann J, Heinemeyer J, Perales M, Zabaleta E, Boekema E, Braun HP. Carbonic anhydrase subunits form a matrix-exposed domain attached to the membrane arm of mitochondrial complex I in plants. J. Biol. Chem. 2006;281:6482-6488.

Tonge R, Shaw J, Middleton B, Rowlinson R, Rayner S, Young J, Pognan F, Hawkins E, Currie I, Davison M. Validation and development of fluorescence two-dimensional differential gel electrophoresis proteomics technology. Proteomics 2001;3:377-96.

Ünlü M, Morgan ME, Minden JS. Difference gel electrophoresis: a single gel method for detecting changes in protein extracts. Electrophoresis 1997;18:2071-2077.

Werhahn W, Braun HP. Biochemical dissection of the mitochondrial proteome from Arabidopsis thaliana by three-dimensional gel electrophoresis. Electrophoresis 2002;23: 640-646

Werhahn W, Niemeyer A, Jänsch L, Kruft V, Schmitz UK, Braun HP. Purification and characterization of the preprotein translocase of the outer mitochondrial membrane from Arabidopsis thaliana: identification of multiple forms of TOM20. Plant Physiol 2001;125:943-954

Wittig, I., Braun, H.P. and Schägger, H. Blue-native PAGE. NATURE Protocols 2006;1:418428.

Wittig I, Karas M, Schägger H. High resolution clear native electrophoresis for in-gel functional assays and fluorescence studies of membrane protein complexes. Mol Cell Proteomics 2007;6:1215-1225. 


\section{Figure legends}

Figure 1: Comparative analysis of the mitochondrial proteomes of Arabidopsis Col-O and Col-O- $\Delta$ Atlg47260. Proteins of the Col-O fraction were pre-labelled by Cy3, proteins of the Col-O- $\Delta$ Atlg47260 fraction by Cy5. Combined protein fractions were separated by 2D Bluenative / SDS-PAGE and protein visualization was carried out by laser scanning at the respective wavelengths using the Typhoon laser scanner. On the resulting overlay image, Cy3 is represented by green and Cy5 by red. Proteins of reduced abundance in the mutant are green, proteins of increased abundance in the mutant are red (do not occur), and proteins of equal abundance in the two compared fractions are yellow. The molecular masses of standard proteins are given to the right of the figure; the identities of protein complexes are given above the gel. I: complex I; V: complex V; $\mathrm{III}_{2}$ : dimeric complex III, I+ $\mathrm{III}_{2}$ : supercomplex containing complex I and dimeric complex III. Reprinted from Perales et al. 2005, with permission from Elsevier.

Figure 2: Heat stability of the mitochondrial OXPHOS complexes from Arabidopsis. Purified mitochondria from Arabidopsis were either heat treated $\left(2\right.$ minutes at $\left.50^{\circ} \mathrm{C}\right)$ or kept on ice for the same time period. Subsequently, organelle fractions were solubilized by dodecylmaltoside and proteins were separated by 2D Blue native / SDS PAGE. A: Coomassie-stained gel of the mitochondrial fraction kept on ice; $\underline{\mathbf{B}}$ : Coomassie-stained gel of the mitochondrial fraction heat treated for two minutes; $\underline{\mathbf{C}}$ : DIGE analysis of a mixed fraction. The mitochondrial fraction kept on ice was pre-labelled by $\mathrm{Cy} 3$, the heat treated mitochondrial fraction was labelled by Cy5. Visualization of CyDyes was carried out by laser scanning at the respective wavelengths using the Typhoon laser scanner. On the resulting overlay image, Cy3 is represented by green and Cy5 by red. Protein complexes destabilized by the heat treatment are green, protein complexes stable in the presence of the heat treatment are yellow. The molecular masses of standard proteins are given to the right of the figure; the identities of protein complexes are given above the gels (I: complex I; II: complex II; $\mathrm{III}_{2}$ : dimeric complex III; IV: complex IV; V: complex V). 
Figure 3: Comparison of the mitochondrial proteomes of Arabidopsis and Polytomella. Purified organelle fractions were solubilized with dodecylmaltoside and pre-labelled with Cy5 (Polytomella) or Cy3 (Arabidopsis). Subsequently, protein fractions were mixed and separated by 2D Blue native / SDS PAGE. Visualization of CyDyes was carried out by laser scanning at the respective wavelengths using the Typhoon laser scanner. On the resulting overlay image, Cy3 is represented by green (Arabidopsis) and Cy5 by red (Polytomella). The molecular masses of standard proteins are given to the right of the figure; the identities of protein complexes are given above the gel (protein complexes of Polytomella in red and of Arabidopsis in green). $\mathrm{V}_{2}$ : dimeric ATP synthase; $\left[\mathrm{V}_{2}\right]$ : subcomplex of dimeric ATP synthase; I: complex I; V: complex V; $\mathrm{III}_{2}$ : dimeric ATP synthase; $\mathrm{F}_{1}: \mathrm{F}_{1}$ part of complex V.

Figure 4: Comparison of the mitochondrial and the chloroplast proteomes of Arabidopsis. Purified organelle fractions were solubilized with dodecylmaltoside and pre-labelled with Cy3 (mitochondria) or Cy5 (chloroplasts). Subsequently, protein fractions were mixed and separated by 2D Blue native / SDS PAGE. Visualization of CyDyes was carried out by laser scanning at the respective wavelengths using the Typhoon laser scanner. On the resulting overlay image, $\mathrm{Cy} 3$ is represented by green (mitochondria) and $\mathrm{Cy} 5$ by red (chloroplast). The molecular masses of standard proteins are given to the right of the figure; the identities of protein complexes are given above the gel. PSII $_{2}+\mathrm{LCII}$ : photosystem II supercomplex + light harvesting complex II; PSI: photosystem I; $b_{6}$ f: cytochrome $b_{6} f$ complex; LCII: light harvesting complex II trimer; I: complex I; V: ATP synthase; $\mathrm{III}_{2}$ : dimeric complex III, $\mathrm{F}_{1}$ : $\mathrm{F}_{1}$ part of ATP synthase.

Figure 5: Principle of three-dimensional analysis of a defined protein fraction using the DIGE system. Protein fractions (e.g. purified organelles) are solubilized using a non-ionic detergent and protein complexes are separated by one-dimensional Blue-native PAGE. Two protein complexes are subsequently electroeluted and labelled with different CyDyes. The whole proteome to be analyzed is labelled by a third CyDye. Finally, all 3 fractions are mixed and co-electrophoresed on a 2D IEF / SDS gel. Visualization of pre-labelled proteins is carried out by laser scanning at the respective wavelengths using the Typhoon laser scanner. 
Figure 6: Mapping of mitochondrial protein complexes from Arabidopsis by threedimensional Blue native / IEF / SDS PAGE. Purified organelles were solubilized by digitonin and protein complexes were separated by one-dimensional Blue native PAGE. The NADH dehydrogenase complex (complex I) and the ATP Synthase complex (complex V) were electroeluted and pre-labelled with Cy2 (complex I) and Cy3 (complex V). A fraction representing total mitochondrial protein was pre-labelled with $\mathrm{Cy} 5$. The three fractions were mixed and co-electrophoresed on an IEF / SDS gel. Visualization of CyDyes was carried out by laser scanning at the respective wavelengths using the Typhoon laser scanner. On the resulting overlay image, the total mitochondrial proteome is given in red, the subunits of complex V in green and the subunits of complex I in blue. The molecular masses of standard proteins are given to the right of the figure. 


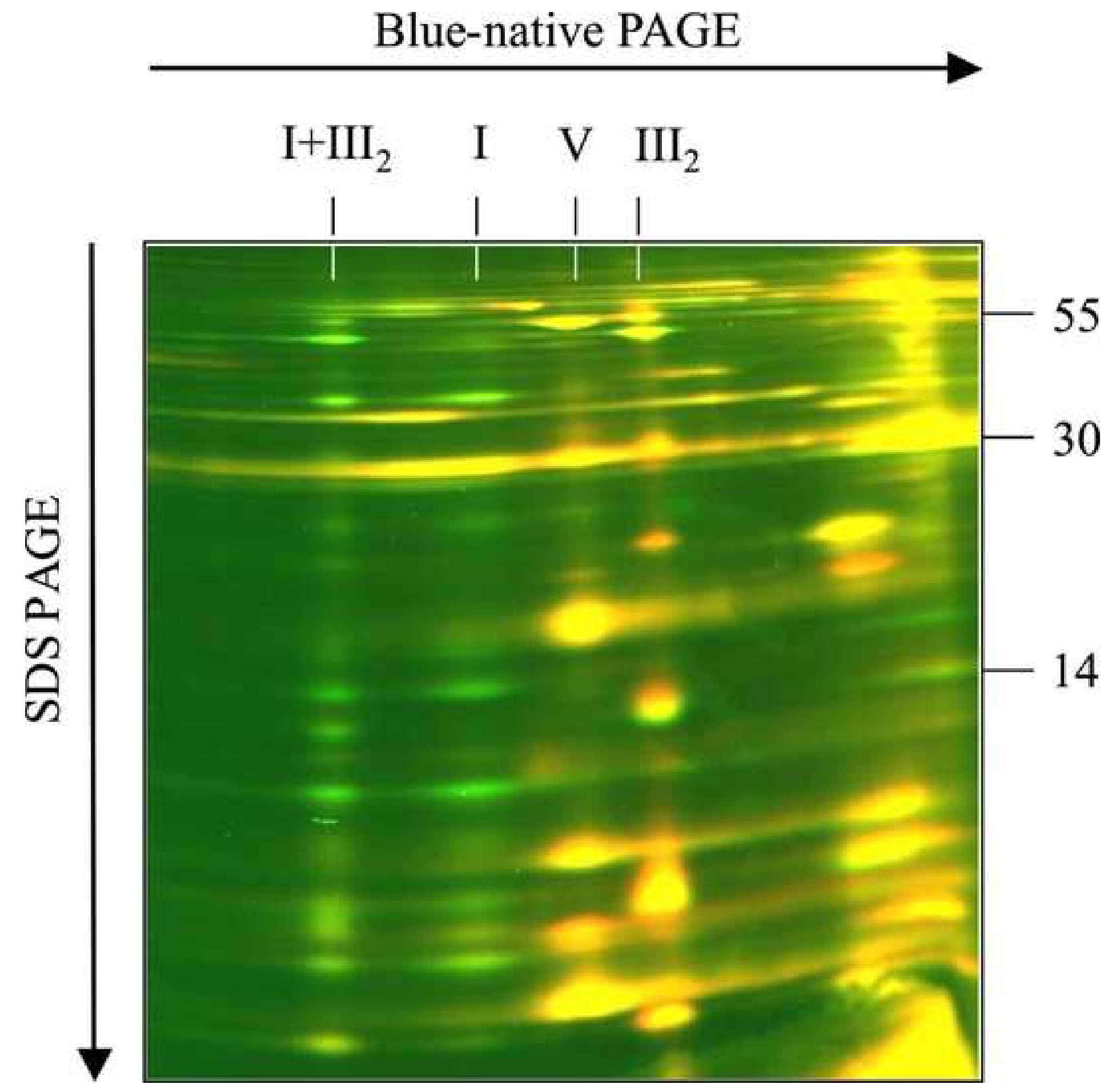



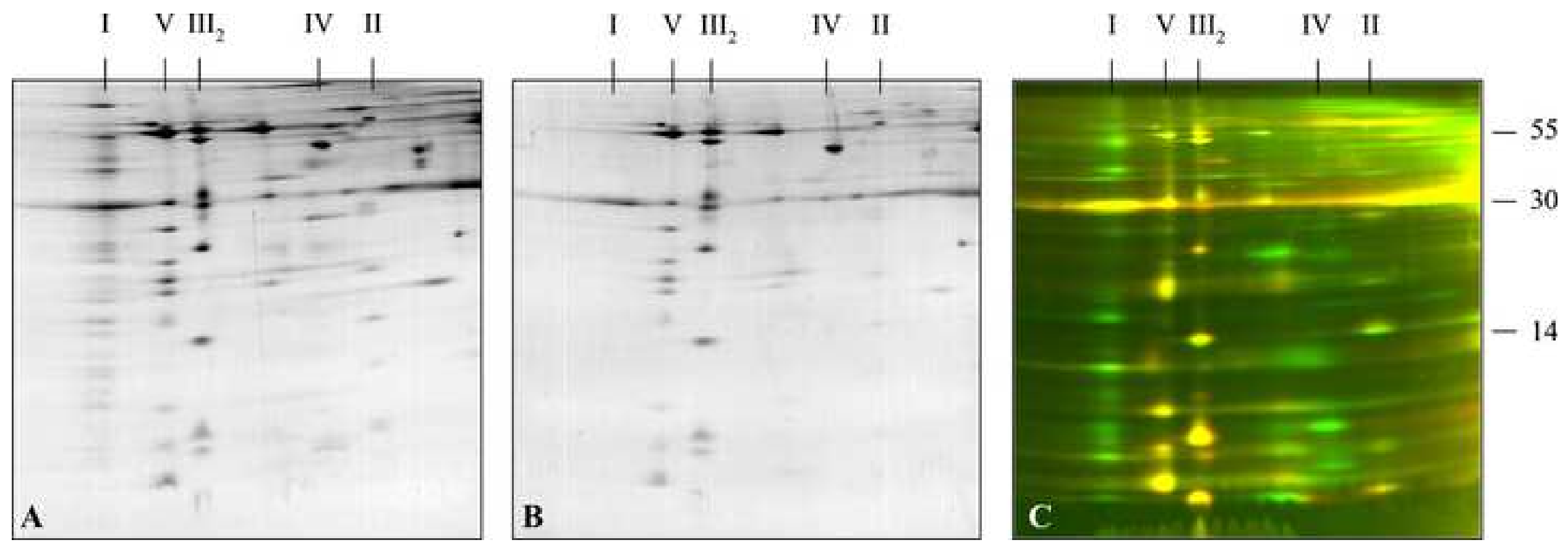
Click here to download high resolution image
Clice 3

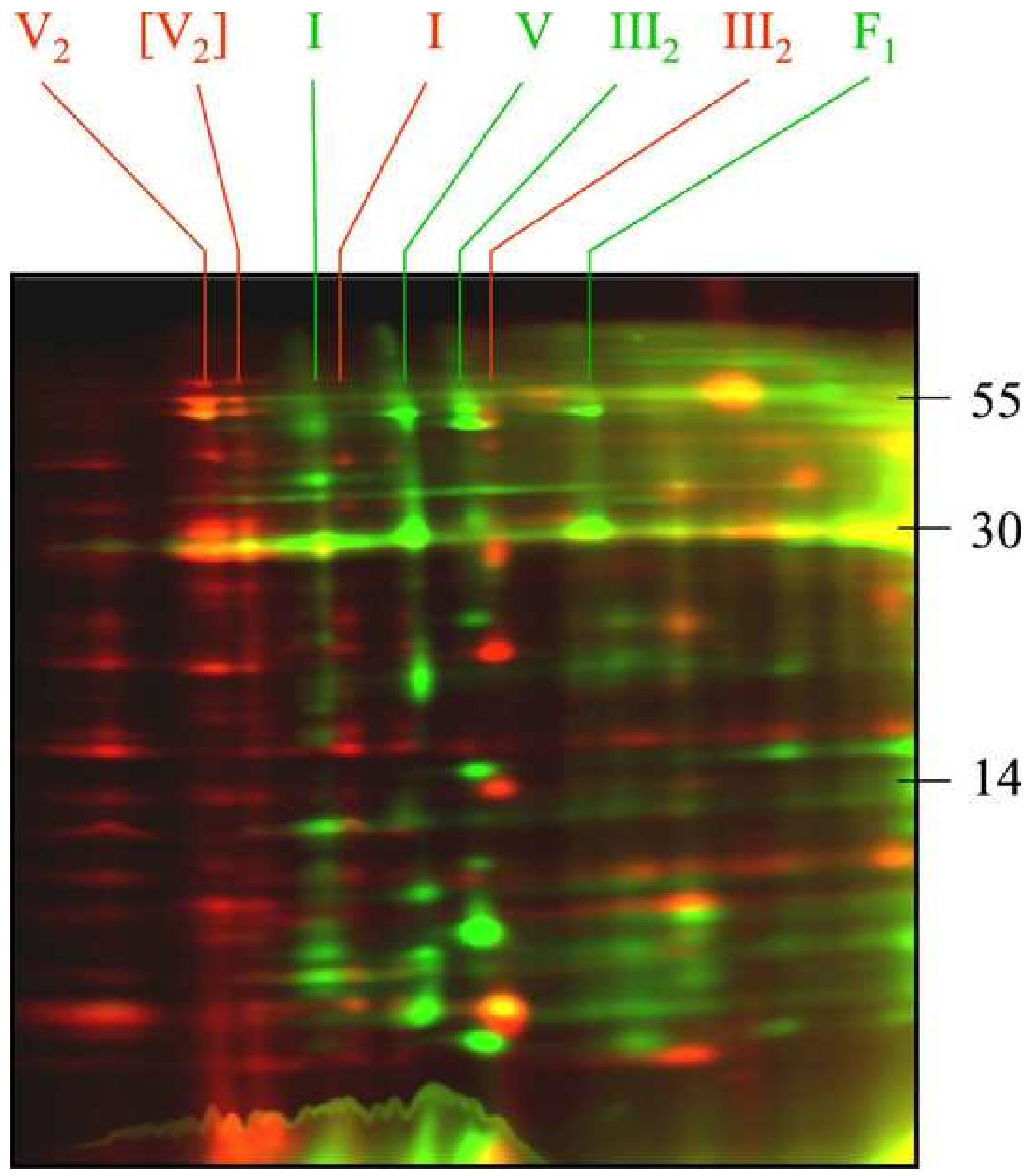


Figure 4
Click here to download high resolution image

\section{PSII $_{2}+$ LCII I V PSI $\quad$ III $_{2} \quad \mathrm{~F}_{1} \mathrm{~F}_{1} / \mathrm{b}_{6} \mathrm{f} \quad$ LCII}

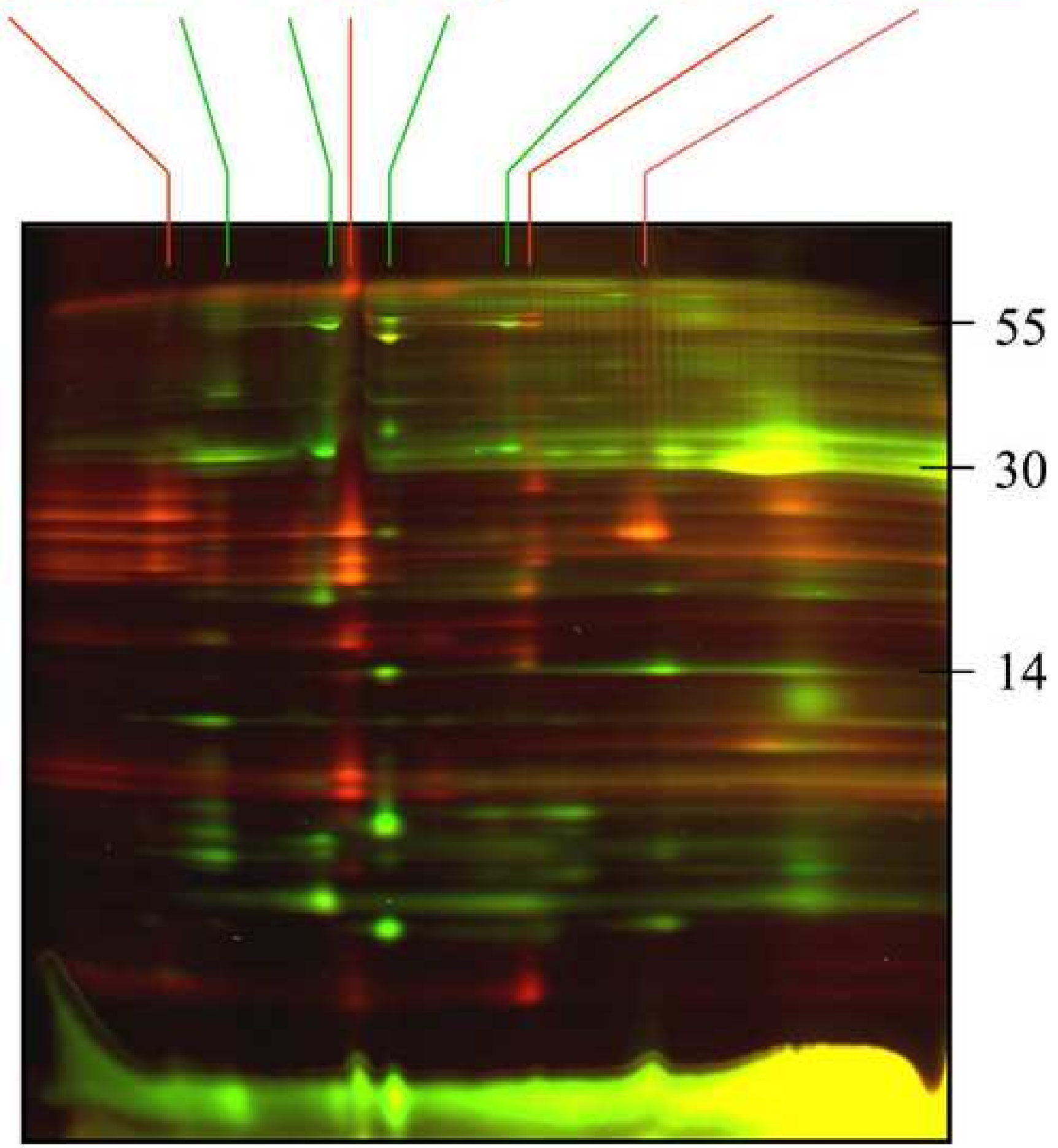




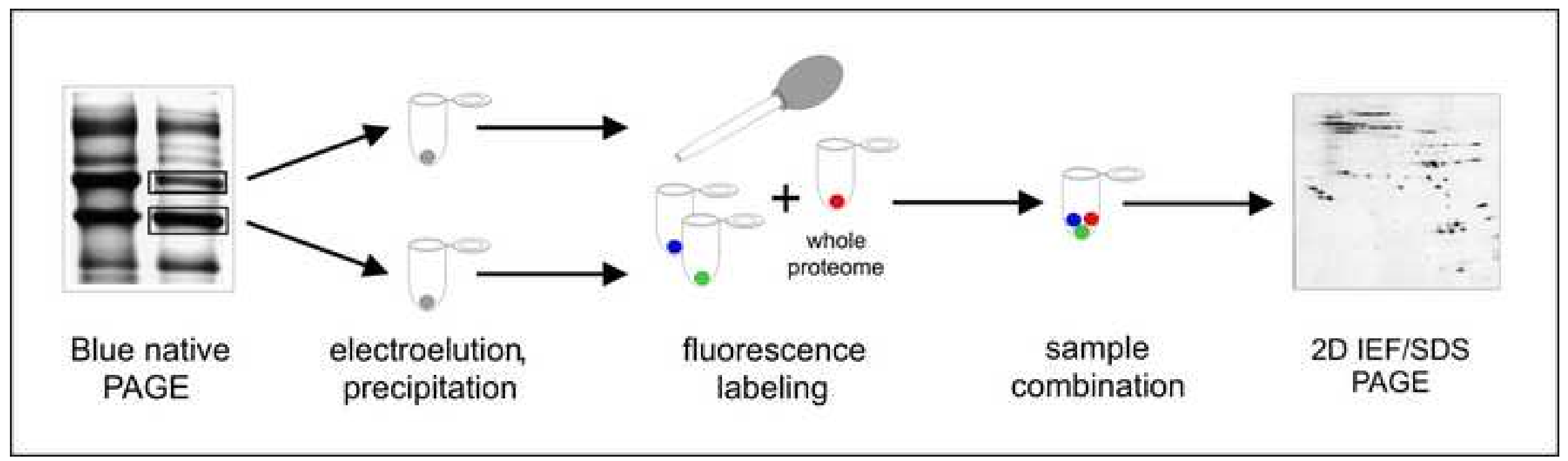




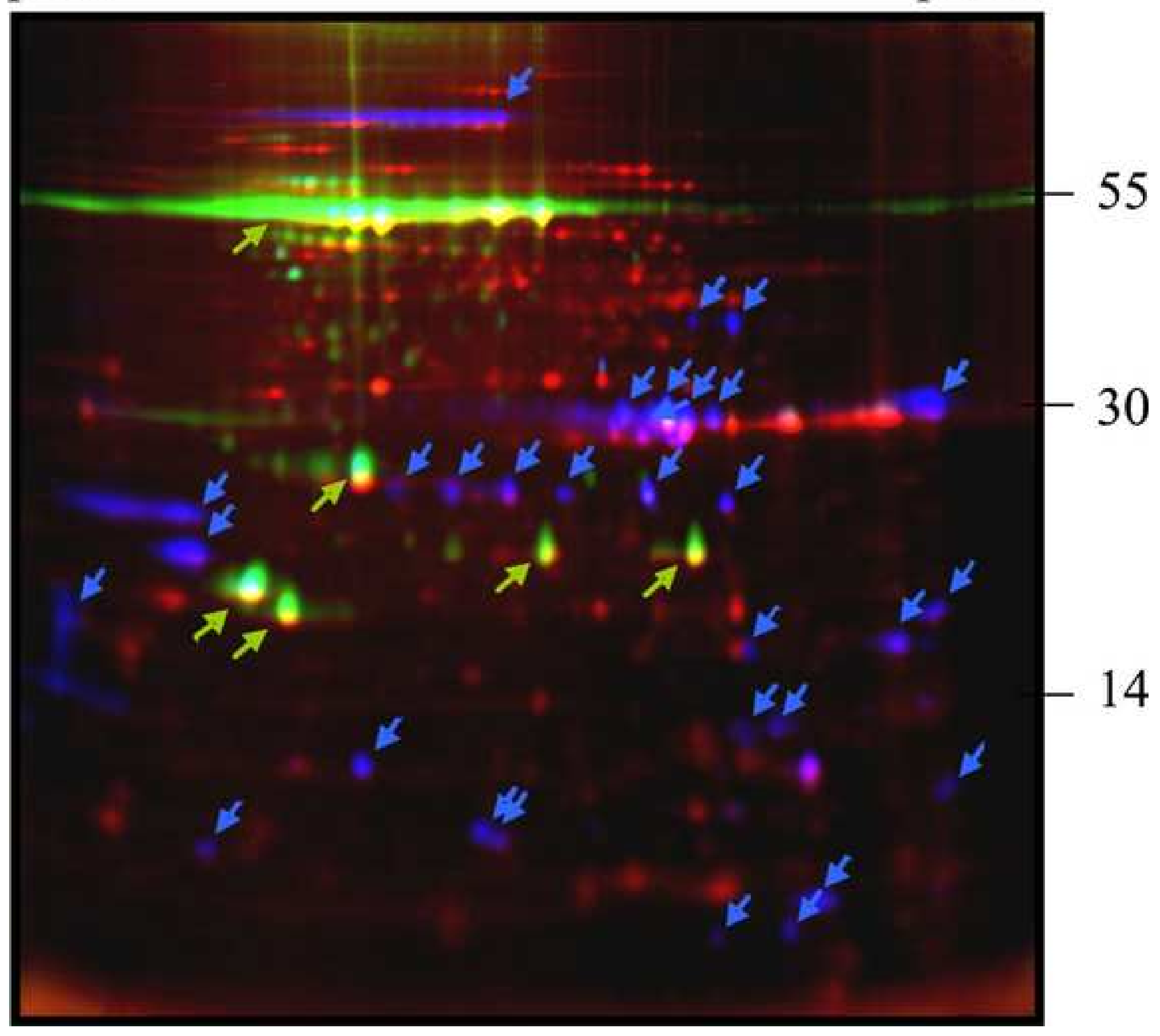

